\title{
The surgical Treatment and Outcome of Pathological Fracture in Patients with Giant Cell Tumor of Bone
}

\author{
Tomoaki Torigoe ${ }^{1}$, Atsuhiko Terakado ${ }^{1}$, Yoshiyuki Suehara ${ }^{1}$, Taketo Okubo ${ }^{1}$, Tatsuya Takagi ${ }^{1}$, \\ Kazuo Kaneko ${ }^{1}$, Yasuo Yazawa ${ }^{2}$ \\ ${ }^{1}$ Department of Orthopaedic Surgery, Juntendo University School of Medicine, Tokyo, Japan \\ ${ }^{2}$ Department of Orthopaedic Oncology, Saitama International Medical Center, Saitama, Japan \\ E-mail: ttorigoe@juntendo.ac.jp \\ Received April 12, 2011; revised June 13, 2011; accepted June 24, 2011
}

\begin{abstract}
Between 1992 and 2008, we treated 35 patients with giant cell tumor (GCT) of bone, seven of the 35 presented with a pathological fracture. The fractures were located in the femur in five, and in the humerus and radius in one patient each. The surgical treatments were curettage in six cases and wide resection in the distal radius case. Two of the seven patients developed local recurrence, giving a local recurrence rate of $29 \%$. The local recurrence rate in GCT patients without a pathological fracture was $21 \%$. There was a tendency for there to be a higher recurrence rate associated with fractured GCT, but no statistically significant difference was observed between the two groups. Therefore, it was considered that a pathological fracture was not a risk factor for local recurrence in GCT.
\end{abstract}

Keywords: Giant Cell Tumor of Bone, Pathological Fracture

\section{Introduction}

There have been several reports that a pathological fracture in subjects with a primary malignant bone tumor is a risk factor for local recurrence and survival [1]. Scully reported that pathological fracture was a statistically risk factor for local recurrence in osteosarcoma [2]. Although a giant cell tumor (GCT) of bone is considered to have the potential for local recurrence [3], it is unclear whether a pathological fracture is a risk factor for local recurrence in GCT cases. We studied the relationship between pathological fractures and local recurrence in GCT cases.

\section{Patients and Methods}

We retrospectively reviewed 35 patients with GCT in the extremities treated at Juntendo University Hospital and Juntendo Orthopedic Oncology Collaboration (JOOC) affiliated hospitals between 1992 and 2008. The clinical and radiological records of all 35 patients were reviewed. Clinical and radiological evidence of a fracture had been seen either at diagnosis or during the course of preoperative treatment. A histopathological diagnosis was ob- tained in all of the cases. Statistical comparisons were made using a Chi-squared analysis and the Fisher exact test.

\section{Results}

In total, seven of 35 patients had a pathological fracture before the initial surgery, four of whom were male, and 3 were female, and they had a median age of 25 years (18 to 36) (Table 1). The median follow-up was 35 months ( 5 to 73 ). The tumor was located in the femur in five, and in the humerus and radius in one patient each. In the femur fractures, four were distal, and one was proximal. In the humerus, the fracture was in the proximal humerus, and in the radius, the fracture was in the distal portion.

The fractures had been present at diagnosis in six patients, and developed after biopsy in one patient. Six fractures occurred after trauma and one occurred spontaneously. According to the grading system of Campanacci, two lesions were grade I, two were grade II, and three were grade III. The surgical treatment was curettage of the tumor with high speed burring in six, and wide resection with a vascularized fibula graft in one distal radius case. Chemical adjuvant treatment was indicated in five 
Table 1. Summary of seven patients with GCT with pathological fracture.

\begin{tabular}{|c|c|c|c|c|c|c|c|c|}
\hline Case & Age/Sex & Location* & $\begin{array}{c}\text { Campanacci } \\
\text { grade }\end{array}$ & Surgery & Adjuvant & Reconstruction & Local Recurrence & \\
\hline \multirow[t]{3}{*}{1} & $22 / \mathrm{M}$ & D Femur & II & $1^{\text {st }}$ Curettage & Alcohol & $1^{\text {st }}$ Bone graft + PMMA & $(+)$ & 73 \\
\hline & & & & $2^{\text {nd }}$ Wide resection & None & $2^{\text {nd }}$ Prosthesis & $(+)$ & \\
\hline & & & & $3^{\text {rd }}$ Wide resection & None & $3^{\text {rd }}$ None & $(-)$ & \\
\hline \multirow[t]{2}{*}{2} & $21 / \mathrm{F}$ & D Femur & III & $1^{\text {st }}$ Curettage & Alcohol & $1^{\text {st }}$ Bone graft + PMMA & $(+)$ & 61 \\
\hline & & & & $2^{\text {nd }}$ Curettage & Alcohol & $2^{\text {nd }}$ Bone graft + PMMA & $(-)$ & \\
\hline 3 & $19 / F$ & D Femur & II & Curettage & $\begin{array}{l}\text { Alcohol + } \\
\text { Phenol }\end{array}$ & Bone graft & $(-)$ & 24 \\
\hline 4 & $36 / F$ & D Femur & I & Curettage & None & Bone graft & $(-)$ & 73 \\
\hline 5 & $23 / \mathrm{M}$ & P Femur & I & Curettage & Alcohol & Bone graft & $(-)$ & 5 \\
\hline 7 & $18 / \mathrm{M}$ & P Humerus & III & Curettage & Alcohol & Bone graft + PMMA & $(-)$ & 32 \\
\hline
\end{tabular}

*D, Distal; P, Proximal; PMMA, Polymethylmethacrylate.

of six curettage cases. Local recurrence developed in two distal femur cases at 11 and 21 months after surgery.

The Campanacci grades in the local recurrent cases was grade II and III. The treatment of the local recurrence was repeat curettage in the patient with the grade III tumor, and wide resection and reconstruction with an endoprosthesis in the patient with the grade II tumor. The patient treated with a wide resection developed a second local recurrence in the soft tissue 62 months after the second operation. This patient was treated with a wide resection of the recurrent tumor in the soft tissue, and no third recurrence has been observed as of the most recent follow-up, which was 69 months after the third operation.

A total of 28 of the 35 GCT cases without fractures were studied as controls. The tumors without fractures were located in the femur in nine, in the tibia in ten, in the radius in five, and in the fibula in four. The Campanacci grades of these tumors were evaluated as grade I in 14 cases, II in four and III in ten cases. The surgical treatment of tumors without a fracture was curettage with or without chemical adjuvant in 23 cases, and wide resection in 5 cases. The median age of patients without a fracture was 31 years old (19 to 57) and no statistically significant difference was observed in the age between the groups with and without fractures $(p=0.06)$. There were also no significant differences in other factors, anatomical location, Campanacci grade, surgery or follow-up period between two groups. A local recurrence was observed in six of the 28 cases without fractures. The overall rate of local recurrence in all 35 patients was $23 \%$. The local recurrence rate in the group with fractures was $29 \%(2 / 7)$ and was $21 \%$ in the subjects without fractures (6/28). Although there was a tendency for there to be a higher recurrence rate associated with a patho- logical fracture, no statistically significant difference was observed between the two groups ( $p=0.69$ ). The local recurrence rate after the curettage operation was 33\% (2/6) in cases with a pathological fracture, and was $26 \%$ $(6 / 23)$ in cases without a fracture, which was also not significantly different. Although no distant metastasis was observed in cases with fractures, multiple lung and soft tissue metastases were observed in one of the cases without a fracture.

\section{Discussion}

Although a giant cell tumor of bone is a benign tumor, it is considered to be locally aggressive and has the potential to result in local recurrence, like malignant tumors. The local recurrence rate of GCT varies in the literature, and the prevalence of local recurrence ranges from 0 to $45 \%$ after burring and/or chemical adjuvant treatment [4-8]. The reported risk factor for local recurrence of GCT was tumor progression outside the bone. Campanacci reported a radiographic grading system for GCT, and this grading system designates tumors on a scale from grades I to III [9]. Grade I tumors have a well-defined border with a thin rim of mature bone, and the cortex is intact or slightly thinned, but not deformed in plain X-ray. Grade II tumors have relatively well-defined margins but no radiopaque rim, and the combined cortex and rim of the reactive bone is rather thin and moderately expanded, but still present. Grade III tumors have fuzzy borders suggesting a rapid and possible permeating growth, with the tumor bulging into the soft tissue, but with the soft tissue lesion not following the contour of the bone, and not limited by an apparent shell of reactive bone. In some studies, a correlation between the Campanacci grade and 
recurrence rate was reported [10,11]. Prosser reported that the overall local recurrence rate of GCT was $19 \%$, and the recurrence rate of the tumors with extraskeletal extension (Campanacci Grade III) was 29\% [12].

In osteosarcoma, a primary bone malignancy, Sculley reported that a pathological raised concerns about tumor dissemination by the hematoma, and should be considered to be a contraindication to limb salvage surgery [13]. Abudu reported that limb sparing surgery with adequate margins of excision could be achieved in many patients with pathological fractures due to primary osteosarcoma without compromising survival, but the risk of local recurrence was significant [14]. It is possible that a pathological fracture in GCT may release tumor cells into the surrounding soft tissues like in osteosarcoma, and Cooper reported that GCT tumor cells were observed in the synovium of the ankle joint after a distal tibia pathological fracture and in the soft tissues around a fracture site in the distal femur [15]. Campanacci reported a possibility that a pathological fracture was the risk factor in GCT, but the relationship was not clear in his report [9]. O'donnell reported that the overall local recurrence rate of GCT was $25 \%$, and that the local recurrence rate of GCT in patients with a pathological fracture was 50\%, thus indicating that a pathological fracture was associated with an increased recurrence rate [16]. Conversely, some reports have suggested that there is a lower local recurrence rate in GCT patients with a fracture than in those without a fracture, but the surgeons in this report were more inclined to perform extensive surgery for lesions with pathological fractures, because they considered there was a risk of tumor cell contamination into the surrounding tissues $[8,17]$. Dreinhöfer reported that four of 10 patients with fractured GCT at diagnosis had local recurrence after the curettage surgery, and that the local recurrence rate in patients with fractured GCT was similar to that in patients with non-fractured GCT [18]. He suggested that extensive surgery, such as an en-bloc resection and reconstruction with tumor prosthesis, was not necessary for GCT with pathological fracture as the first operation, and extensive treatment should be considered only when a local recurrence occurs after a curettage surgery.

In our present study, no significant difference was observed in the local recurrence rate between GCT patients with and without fractures. We therefore do not consider a pathological fracture to be a definite risk factor for local recurrence in GCT, and a curettage operation with adjuvant treatment should therefore be advocated for treating such tumors.

\section{References}

[1] J. A. Bramer, A. A. Abudu, R. J. Grimer, S. R. Carter, and R. M. Tillman, "Do Pathological Fractures Influence
Survival and Local Recurrence Rate in Bony Sarcomas?” European Journal of Cancer, Vol. 43, No. 13, September 2007, pp. 1944-1951. doi:10.1016/j.ejca.2007.07.004

[2] S. P. Scully, H. T. Temple, R. J. O’Keefe, H. J. Mankin and M. Gebhardt, "The Surgical Treatment of Patients with Osteosarcoma Who Sustain a Pathologic Fracture,” Clinical Orthopaedics and Related Research, No. 324, March 1996, pp. 227-32. doi:10.1097/00003086-199603000-00028

[3] H. R. Blackley, J. S. Wunder, A. M. Davis, L. M. White, R. Kandel and R. S. Bell, "Treatment of Giant-Cell Tumors of Long Bones with Curettage and Bone-Grafting," Journal of Bone and Joint Surgery American Volume, Vol. 81, No. 6, June 1999, pp. 811-820.

[4] D. C. Dahlin, R. E. Cupps and E. W. Johnson Jr., "Giant-Cell Tumor: A Study of 195 Cases,” Cancer, Vol. 25, No. 5, May 1970, pp. 1061-1070.

doi:10.1002/1097-0142(197005)25:5<1061::AID-CNCR 2820250509>3.0.CO;2-E

[5] F. M. Klenke, D. E. Wenger, C. Y. Inwards, P. S. Rose and F. H. Sim, "Recurrent Giant Cell Tumor of Long Bones: Analysis of Surgical Management,” Clinical Orthopaedics and Related Research, No. 469, April 2011, pp. 1181-1187. doi:10.1007/s11999-010-1560-9

[6] F. Vult von Steyern, H. C. Bauer, C. Trovik, A. Kivioja, P. Bergh, P. Holmberg Jörgensen, G. Follerås and A. Rydholm, "Treatment of Local Recurrences of Giant Cell Tumour in Long Bones after Curettage and Cementing. A Scandinavian Sarcoma Group Study," Journal of Bone and Joint Surgery British Volume, Vol. 88, No. 4, April 2006, pp. 531-535. doi:10.1302/0301-620X.88B4.17407

[7] P. J. McGrath, "Giant-Cell Tumour of Bone: An Analysis of Fifty-Two Cases,” Journal of Bone and Joint Surgery British Volume, Vol. 54, No. 2, May 1972, pp. 216-229.

[8] S. E. Larsson, R. Lorentzon and L. Boquist, "Giant-Cell Tumor of Bone. A Demographic, Clinical, and Histopathological Study of All Cases Recorded in the Swedish Cancer Registry for the Years 1958 through 1968,” Journal of Bone and Joint Surgery American Volume, Vol. 57, No. 2, March 1975, pp. 167-73.

[9] M. Campanacci, N. Baldini, S. Boriani and A. Sudanese, "Giant-Cell Tumor of Bone," Journal of Bone and Joint Surgery American Volume, Vol. 69, No. 1, January 1987, pp. 106-114.

[10] F. M. Klenke, D. E. Wenger, C. Y. Inwards, P. S. Rose and F. H. Sim, "Giant Cell Tumor of bone: Risk Factors for Recurrence," Clinical Orthopaedics and Related Research, Vol. 469, No. 2, February 2011, pp. 591-599. doi:10.1007/s11999-010-1501-7

[11] S. D. Pals and R. M. Wilkins, "Giant Cell Tumor of Bone Treated by Curettage, Cementation, and Bone Grafting," Orthopedics, Vol. 15, No. 6, June 1992, pp. 703-708.

[12] G. H. Prosser, K. G. Baloch, R. M. Tillman, S. R. Carter and R. J. Grimer, "Does Curettage without Adjuvant Therapy Provide Low Recurrence Rates in giant-Cell Tumors of Bone?" Clinical Orthopaedics and Related Research, No. 435, June 2005, pp. 211-218. doi:10.1097/01.blo.0000160024.06739.ff 
[13] S. P. Scully, M. A. Ghert, D. Zurakowski, R. C. Thompson and M. C. Gebhardt, "Pathologic Fracture in Osteosarcoma: Prognostic Importance and Treatment Implications", Journal of Bone and Joint Surgery American Volume, Vol. 84, No. 1, January 2002, pp. 49-57.

[14] A. Abudu, N. K. Sferopoulos, R. M. Tillman, S. R. Carter and R. J. Grimer, "The Surgical Treatment and Outcome of Pathological Fractures in Localised Osteosarcoma,” Journal of Bone and Joint Surgery British Volume, Vol. 78, No. 5, September 1996, pp. 694-698.

[15] K. L. Cooper, J. W. Beabout and D. C. Dahlin, "Giant Cell Tumor: Ossification in Soft-Tissue Implants,” Radiology, Vol. 153, No. 3, December 1984, pp. 597-602.

[16] R. J. O’Donnell, D. S. Springfield, H. K. Motwani, J. E.
Ready, M. C. Gebhardt and H. J. Mankin, "Recurrence of Giant-Cell Tumors of the Long Bones after Curettage and Packing with Cement," Journal of Bone and Joint Surgery American Volume, Vol. 76, No. 12, December 1994, pp. 1827-1833.

[17] D. J. McDonald, F. H. Sim, R. A. McLeod and D. C. Dahlin, "Giant-Cell Tumor of Bone," Journal of Bone and Joint Surgery American Volume, Vol. 68, No. 2, February 1986, pp. 235-242.

[18] K. E. Dreinhöfer, A. Rydholm, H. C. Bauer and A. Kreicbergs, "Giant-Cell Tumours with Fracture at Diagnosis. Curettage and Acrylic Cementing in Ten Cases,” Journal of Bone and Joint Surgery Britihs Volume, Vol. 77, No. 2, March 1995, pp. 189-193. 